\title{
BI-DIRECTIONAL SWITCHING OF MICRODROPLET ADHESION ON DOPED POLYPYRROLE MICROSTRUCTURES
}

\author{
W. Xu, X. Li, A. Palumbo, C.-H. Choi, and E.-H. Yang \\ Stevens Institute of Technology, Hoboken, New Jersey, USA
}

\begin{abstract}
This paper presents a study of bi-directional switchable adhesion of an organic microdroplet on dodecylbenzenesulfonate doped polypyrrole (PPy(DBS)) microstructures at ultra-low voltages $(0.9 \mathrm{~V})$ in an aqueous environment. The PPy(DBS) switched between an oxidized and a reduced state upon application of low voltages. The PPy(DBS) microstructure oxidized at $0.6 \mathrm{~V}$ behaved like a super sticky surface in regard to an organic droplet; in contrast, the $\mathrm{PPy}(\mathrm{DBS})$ microstructure reduced at $-0.9 \mathrm{~V}$ was super slippery. Utilizing the tunable adhesion property of PPy(DBS) microstructures, we demonstrated a capture/release manipulation of an oil-based dichloromethane (DCM) microdroplet at a voltage of $0.9 \mathrm{~V}$.
\end{abstract}

\section{INTRODUCTION}

The manipulation of microdroplets has broad applications in microfluidics, biochemistry, and lab-on-chip devices [1-3]. Surfaces with tunable adhesion have been studied for the manipulation of microdroplets through external stimuli, such as light, magnetic field, mechanical force, or electrical field [4-8]. Recently, conjugated polymers have attracted a lot of interest due to their tunable wetting properties, which can be induced by a low voltage [9-11]. For example, dodecylbenzenesulfonate doped polypyrrole (PPy(DBS)) exhibits tunable surface wetting property upon application of the ultra-low reduction and oxidation voltages $(\sim 0.9 \mathrm{~V})$. During the redox process, the change of surface wetting property of PPy(DBS) from hydrophobic to hydrophilic is caused by the reorientation of the surfactant dopant molecules (i.e., DBS) in PPy(DBS) (Figure 1) [12].
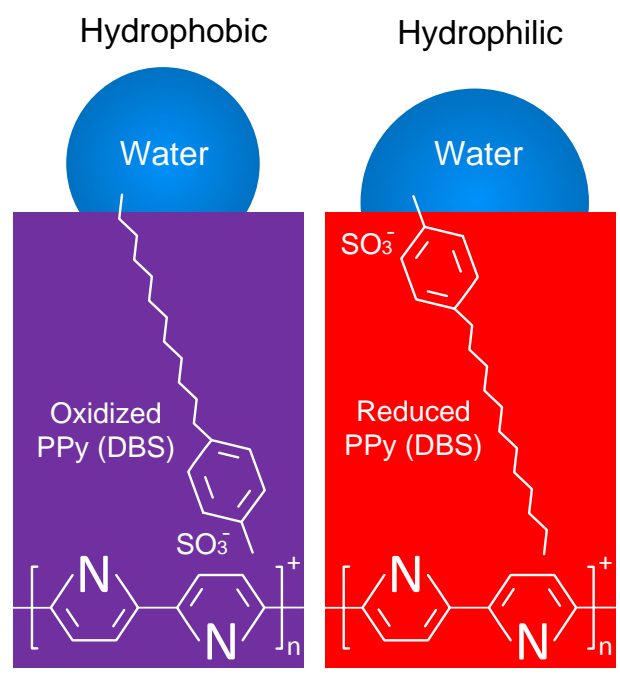

Figure 1: The surface state of PPy(DBS) switched between hydrophilic and hydrophobic during the redox processes initiated by low voltage application. Due to the reorientation of the surfactant dopant molecules in PPy (DBS), oxidized PPy $(D B S)$ is hydrophobic and reduced $P P y(D B S)$ is hydrophilic. The surface adhesion of $P P y(D B S)$ also changes between an oxidized and a reduced state with the change of surface wetting property.
The surface adhesion of conjugated polymers also changes between an oxidized and a reduced state with the change of surface wetting property. Ding et al. have utilized polyaniline nanowire films with potential-modulated tunable adhesion for the transport of an oil droplet by a capture/release process [13]. However, the release of a microdroplet from the surface is still an issue to overcome without the contact of microdroplet with another more adhesive surface. In this work, we demonstrate a reversible switch of surface adhesion, from super sticky to super slippery, by using micropillar patterned PPy(DBS) surfaces, which enables an independent capture and release of microdroplets.

\section{EXPERIMENTAL METHODS}

Micropillar patterned PPy(DBS) samples were fabricated by electropolymerization on $\mathrm{Au} / \mathrm{Cr}$ coated microstructured silicon substrates. The silicon substrates were micropatterned through a photolithography process followed by a deep reactive ion etching (DRIE). The micropatterned silicon substrates were then coated with $\mathrm{Cr}(100 \mathrm{~nm})$ and $\mathrm{Au}(100 \mathrm{~nm})$ by using e-beam evaporation (PVD 75, Kurt Lesker). During the electropolymerization of $\mathrm{PPy}(\mathrm{DBS})$, the $\mathrm{Cr} / \mathrm{Au}$ coated silicon substrates acted as working electrodes and were submerged in an aqueous pyrrole solution consisting of $0.1 \mathrm{M}$ pyrrole (Aldrich) and $0.1 \mathrm{M}$ sodium dodecylbenzenesulfonate (NaDBS) (Aldrich). A saturated calomel electrode (SCE) (Fisher Scientific Inc.) and an Au coated silicon wafer were also submerged in the solution as reference and counter electrodes. The deposition of PPy(DBS) on the Au surface was performed at $0.8 \mathrm{~V}$ versus SCE by using a potentiostat (263A, Princeton Applied Research, Oak Ridge, TN) [11]. The coating thickness was controlled with coating time.

After the microstructured PPy(DBS) samples were prepared, we first studied the change in the surface adhesion of microstructured PPy(DBS) surfaces upon application of reductive and oxidative potentials in a two-electrode system. The PPy(DBS) coated substrate was used as a working electrode and copper tape was connected as the counter electrode. A potential was applied to the PPy(DBS) substrate for the reduction $(-0.9 \mathrm{~V})$ and oxidation $(+0.6 \mathrm{~V})$ reactions. After either reduction or oxidation, the change in the surface adhesion of the PPy(DBS) microstructured surface was analyzed according to the sliding angle of a dichloromethane (DCM) microdroplet in an aqueous environment $\left(0.1 \mathrm{M} \mathrm{NaNO}_{3}\right)$ by using a goniometer system (Model 500, Ramé-hart instrument co., Netcong, NJ, USA).

We then investigated the capture/transport/release process of a micro-liter DCM droplet on the micropatterned PPy(DBS) surface in an aqueous electrolyte environment $\left(0.1 \mathrm{M} \mathrm{NaNO}_{3}\right)$. A droplet of DCM was placed on a glass substrate in a $0.1 \mathrm{M} \mathrm{NaNO}_{3}$ solution. The PPy(DBS) microstructure was submerged in the solution and oxidized first by applying a positive potential (e.g., $0.6 \mathrm{~V}$ ). Then, the PPy(DBS) microstructure was placed in contact with the DCM microdroplet in order to capture it. Once transported to the desired location, a potential of $-0.9 \mathrm{~V}$ was then applied to the PPy(DBS) microstructure in order to release the captured microdroplet. The entire capture, transport, and release process was monitored with the goniometer system. 


\section{RESULTS AND DISCUSSION}

Figure 2 shows the fabricated micropillar patterned PPy(DBS). Figure $2 \mathrm{a}$ is the $\mathrm{Au} / \mathrm{Cr}$ coated silicon substrate with micropillar patterns before PPy(DBS) coating. The diameter and height of the $\mathrm{Au} / \mathrm{Cr}$ coated micropillars are approximately $6 \mu \mathrm{m}$ and $5 \mu \mathrm{m}$, respectively. The $\mathrm{Au}$ and $\mathrm{Cr}$ layers are each $100 \mathrm{~nm}$ thick. Figure $2 \mathrm{~b}$ shows the micropatterned substrate coated with PPy(DBS) through electropolymerization. As shown, the $\mathrm{PPy}(\mathrm{DBS})$ is uniformly coated on the micropillar structures. The thickness of the PPy(DBS) layer is approximately $1 \mu \mathrm{m}$, and the diameter of the PPy(DBS) coated micropillars is approximately $8 \mu \mathrm{m}$.

(a)

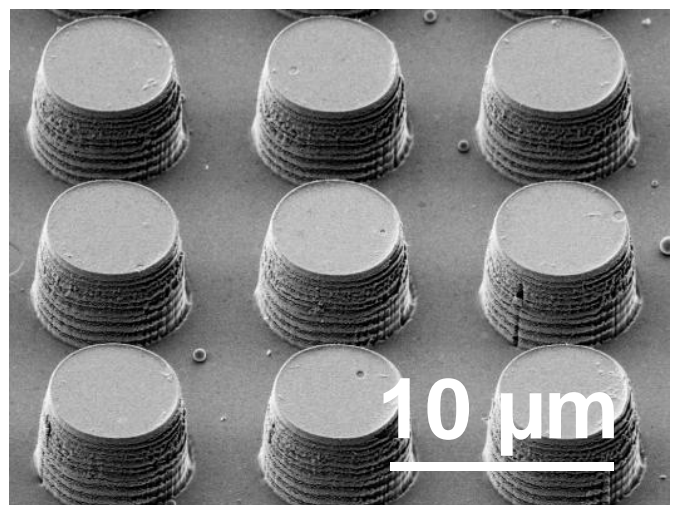

(b)

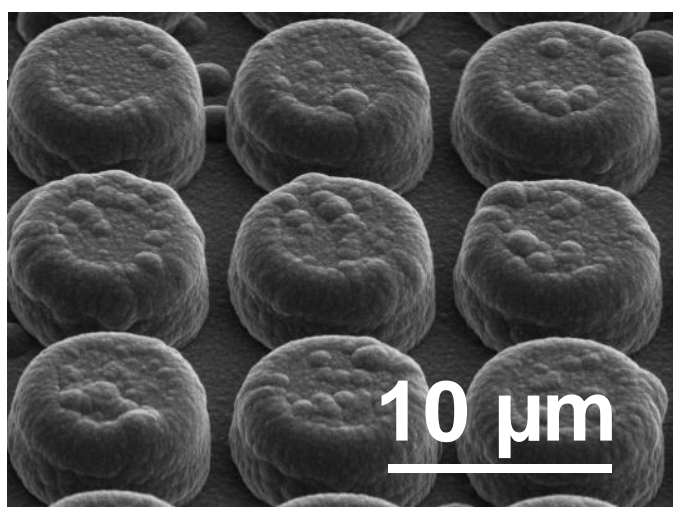

(c)

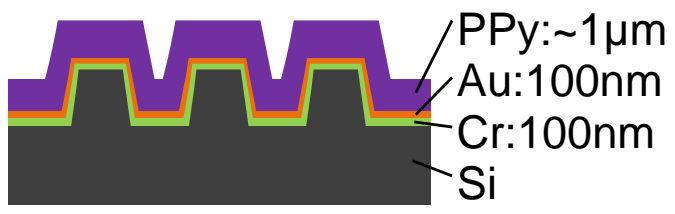

Figure 2: (a) The Au/Cr coated micropatterned silicon substrate used for the electrodeposition of PPy(DBS). (b) The PPy(DBS) microstructures fabricated by using the substrate shown in (a). (c) The Au and Cr layers are each $100 \mathrm{~nm}$ thick, and the thickness of the $P P y(D B S)$ coating is approximately $1 \mu m$.

Figure 3 shows the tunable adhesion property of oil-based dichloromethane (DCM) microdroplets on the PPy(DBS) microstructure in either the oxidized or reduced state. The PPy(DBS) microstructure oxidized at $+0.6 \mathrm{~V}$ behaved like a super sticky surface in regard to the DCM droplet even when the substrate was tilted 90 degrees in an aqueous environment $\left(0.1 \mathrm{M} \mathrm{NaNO}_{3}\right.$ solution). Upon reduction at $-0.9 \mathrm{~V}$, the DCM droplet easily slid off the substrate even with a tilting angle as small as 0.5 degree. These results indicate that the reduced $\mathrm{PPy}(\mathrm{DBS})$ microstructures provide a strongly amplified switchable adhesion between super sticky and super slippery.

(a) Oxidized State

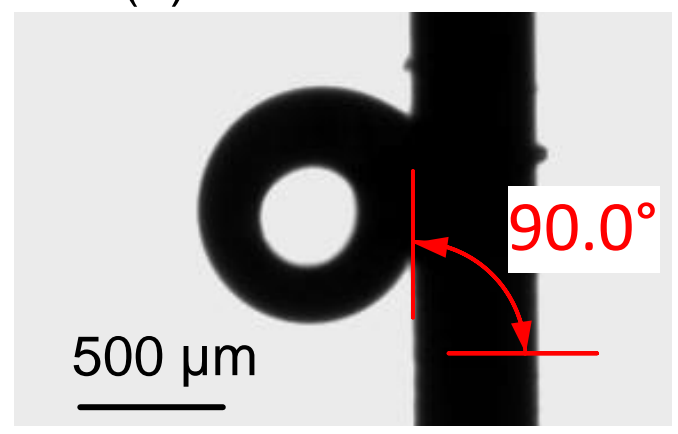

(b) Reduced State

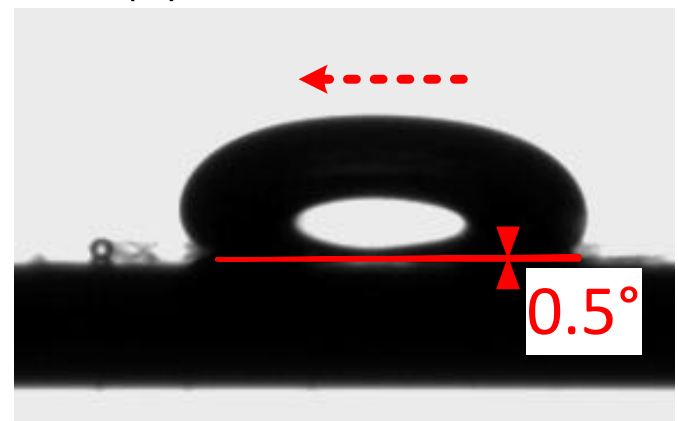

Figure 3: Sliding angles of DCM microdroplets on PPy (DBS) microstructures. (a) When the PPy(DBS) microstructure was oxidized at $+0.6 \mathrm{~V}$, the DCM microdroplet was pinned even when the substrate was tilted to 90 degrees. (b) When PPy(DBS) microstructure was reduced at $-0.9 \mathrm{~V}$, the microdroplet easily slipped off the surface.

Utilizing this switchable adhesion property, we demonstrated a capture/transport/release process of an organic microdroplet at ultra-low voltages in an aqueous environment. The droplet manipulation mechanism is shown in Figure 4. The PPy(DBS) microstructure is first oxidized at a positive potential (e.g., $0.6 \mathrm{~V})$ to capture the organic microdroplet since the oxidized PPy(DBS) behaves a super sticky surface. The PPy(DBS) substrate containing the captured microdroplet is then transported to the desired location (Figure 4b). At this location, a negative potential (e.g, -0.9 V) is then applied to the PPy(DBS) microstructure (Figure 4c). Since the reduced $\mathrm{PPy}(\mathrm{DBS})$ microstructure behaves like a super slippery surface in regard to the organic droplet, the captured microdroplet is released from the substrate by gravity.

Figure 5 shows a demonstration of droplet capture, transport, and release using the PPy(DBS) microstructure. The PPy(DBS) microstructure was first oxidized at $+0.6 \mathrm{~V}$ before being placed in contact with a DCM microdroplet (Figure 5a). Once in contact, the droplet was immediately attached to the oxidized PPy(DBS) microstructure due to a strong adhesion force (Figure $5 \mathrm{~b}$ ) and lifted up from the glass substrate (Figure $5 \mathrm{c}$ ). After the transportation (Figure 5d), the DCM microdroplet was released from the PPy(DBS) microstructure upon application of the reductive potential of $-0.9 \mathrm{~V}$ (Figure 5e) and placed at the desired location (Figure 5f). There was no necessity for the microdroplet to contact 
another more adhesive surface to release from the PPy(DBS) microstructure. During this whole process, the DCM microdroplet remained fully intact and there was no residual droplet left on the PPy(DBS) microstructure.

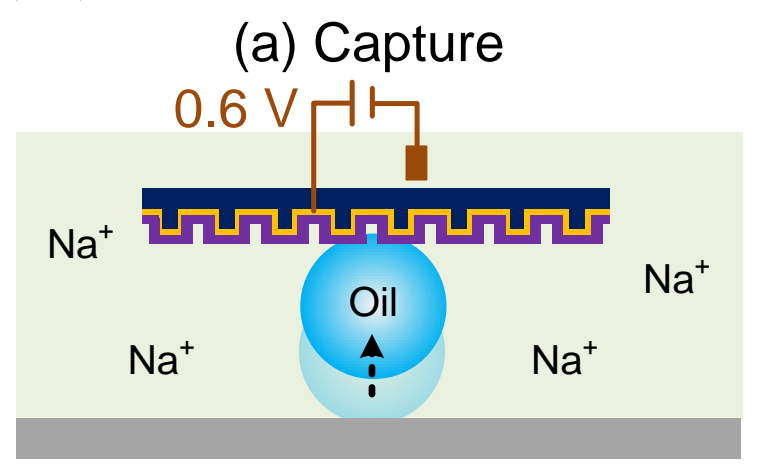

(b) Transport

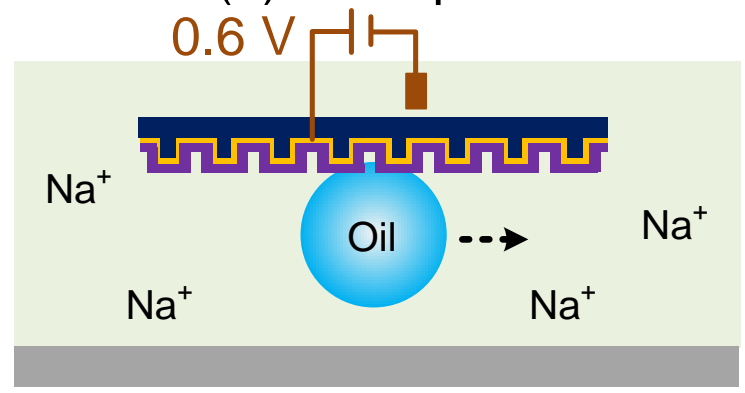

(c) Release

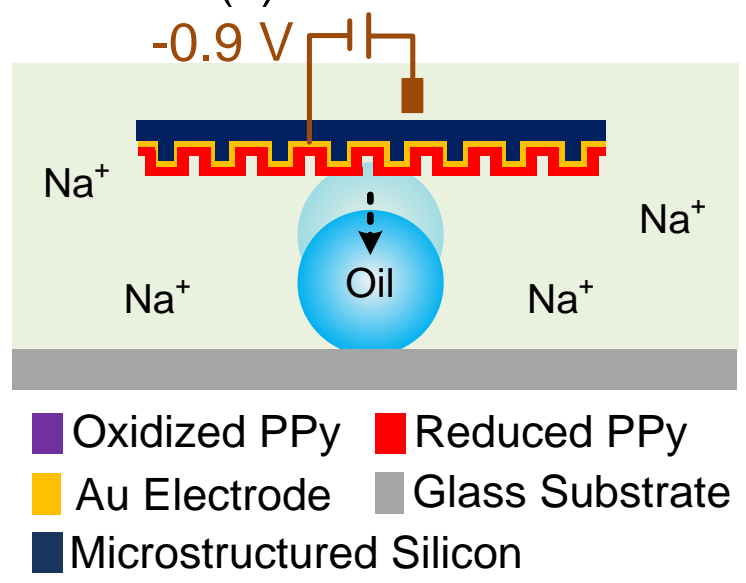

Figure 4: Scheme for the capture and release of an organic droplet in an aqueous electrolyte environment (e.g. $\mathrm{NaNO}_{3}$ solution) utilizing the tunable adhesion of a PPy(DBS) microstructure. (a) The PPy $(D B S)$ microstructure oxidized at $0.6 V$ has a strong adhesion with the organic droplet and is used to capture the microdroplet. (b) Transportation. (c) The reduced PPy(DBS) microstructure, which behaves as a super slippery surface, releases the captured microdroplet after the transportation.

\section{CONCLUSIONS}

In summary, we have demonstrated an ultra-low-voltage controlled capture/release process of organic microdroplets utilizing the tunable adhesion on PPy(DBS) microstructures. This technique has promising applications in microfluidics, antifouling, and oil-water separation.

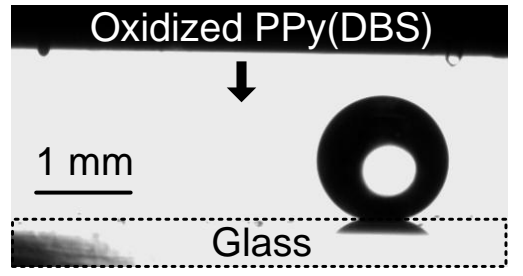

(a) Approaching

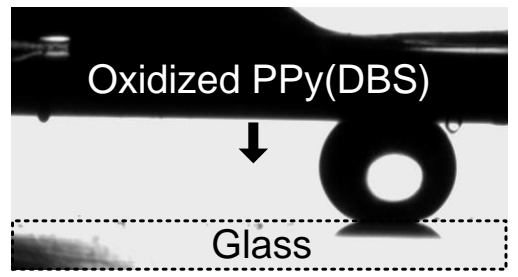

(b) Contacting

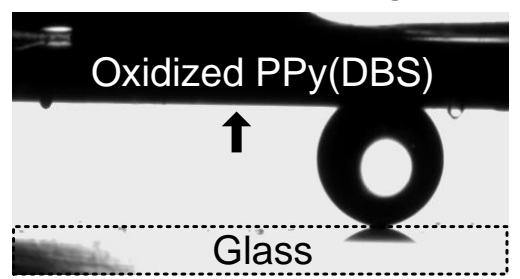

(c) Capturing

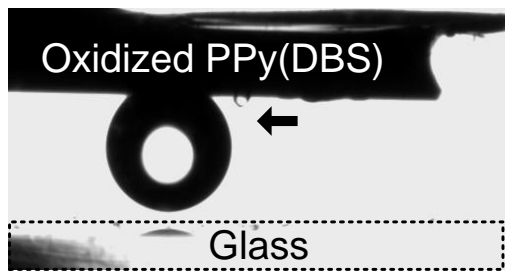

(d) Transporting

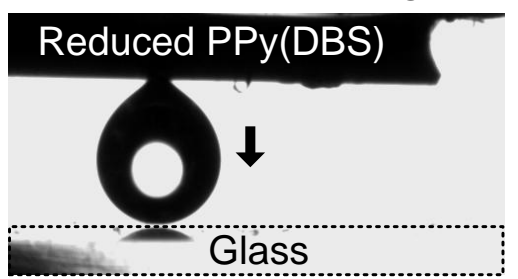

(e) Releasing

Reduced PPy(DBS)

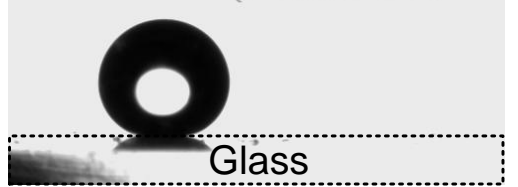

(f) Settling

Figure 5: The process of capturing, transporting and releasing a DCM microdroplet by changing the oxidization state of a micropatterned PPy $(D B S)$ substrate. The DCM microdroplet was captured and transported by the micropatterned PPy $(D B S)$ substrate in an oxidized state and released by reducing the micropatterned PPy substrate. No loss of DCM droplet has been observed during the whole process. 


\section{ACKNOWLEDGEMENTS}

This work has been supported in part by National Science Foundation (EECS-1040007, ECCS-1104870, ECCS-1202269 and EEC-1138244) and Air Force Office for Scientific Research (FA9550-11-1-0272, FA9550-12-1-0326).

\section{REFERENCES}

[1] J.R. Dorvee, A.M. Derfus, S.N. Bhatia, and M.J. Sailor, "Manipulation of Liquid Droplets using Amphiphilic, Magnetic One-Dimensional Photonic Crystal Chaperones", Nature Materials, 3, 896 (2004).

[2] O.D. Velev, B.G. Prevo, and K.H. Bhatt, "On-Chip Manipulation of Free Droplets", Nature, 426, 515 (2003).

[3] V. Srinivasan, V.K. Pamula, and R.B. Fair, "Droplet-Based Microfluidic Lab-on-a-chip for Glucose Detection", Analytica Chimica Acta, 507, 145 (2004).

[4] P.-Y. Chiou, Z.H. Chang, and M.C. Wu, "Droplet Manipulation with Light on Optoelectrowetting Device", Journal of Microelectromechanical Systems , 17, 133 (2008).

[5] D. Wu, S.-Z. Wu, Q.-D. Chen, Y.-L. Zhang, J. Yao, X. Yao, L.-G. Niu, J.-N. Wang, L. Jiang, and H.-B. Sun, "Curvature-Driven Reversible In Situ Switching Between Pinned and Roll-Down Superhydrophobic States for Water Droplet Transportation", Advanced Materials, 23, 545 (2011).

[6] X. Yao, J. Gao, Y. Song, and L. Jiang, "Superoleophobic Surfaces with Controllable Oil Adhesion and Their Application in Oil Transportation", Advanced Functional Materials, 21, 4270 (2011).

[7] X. Zhao, H. Fan, J. Luo, J. Ding, X. Liu, B. Zou, and Y. Feng,
"Electrically Adjustable, Super Adhesive Force of a Superhydrophobic Aligned $\mathrm{MnO}_{2}$ Nanotube Membrane", Advanced Functional Materials, 21, 184 (2011).

[8] X. Hong, X. Gao, and L. Jiang, "Application of Superhydrophobic Surface with High Adhesive Force in No Lost Transport of Superparamagnetic Microdroplet", Journal of the American Chemical Society, 129, 1478 (2007).

[9] E. Smela, "Microfabrication of PPy Microactuators and Other Conjugated Polymer Devices", Journal of Micromechanics and Microengineering, 9, 1 (1999).

[10] Y.-T. Tsai, C.-H. Choi, and E.-H. Yang, "Low-Voltage Manipulation of an Aqueous Droplet in a Microchannel via Tunable Wetting on PPy(DBS)", Lab on a Chip, 13, 302 (2013).

[11] Y.-T. Tsai, C.-H. Choi, N. Gao, and E.-H. Yang, "Tunable Wetting Mechanism of Polypyrrole Surfaces and Low-Voltage Droplet Manipulation via Redox", Langmuir, 27, 4249 (2011).

[12] K.S. Teh, Y. Takahashi, Z. Yao, and Y.-W. Lu, "Influence of Redox-Induced Restructuring of Polypyrrole on its Surface Morphology and Wettability", Sensors and Actuators A: Physical, 155, 113 (2009).

[13] C. Ding, Y. Zhu, M. Liu, L. Feng, M. Wan, and L. Jiang, "PANI Nanowire Film with Underwater Superoleophobicity and Potential-Modulated Tunable Adhesion for No Loss Oil Droplet Transport", Soft Matter, 8, 9064 (2012).

\section{CONTACT}

* E.-H. Yang, tel: +1-201-216-5574; eyang@ @stevens.edu 